\title{
Optimal trading strategy for GenCo in LMP-based and bilateral markets using self-organising hierarchical PSO
}

\author{
C. Boonchuay ${ }^{1}$, W. Ongsakul ${ }^{1}$, J. Zhong ${ }^{2}$, F.F. Wu ${ }^{2}$ \\ ${ }^{I}$ Energy Field of Study, Asian Institute of Technology, THAILAND \\ ${ }^{2}$ Department of Electrical and Electronic Engineering, The University of Hong Kong, CHINA \\ "Corresponding Author: e-mail: ongsakul@ait.asia, Tel +66-2-524-5398, Fax. +66-2-524-5439
}

\begin{abstract}
This paper proposes an optimal trading strategy for a generation company (GenCo) in multi-market environment including day-ahead spot and long term bilateral contract markets using self-organising hierarchical particle swarm optimisation with time-varying acceleration coefficients (SPSO-TVAC). The proposed trading strategy is formulated as a two-stage optimisation problem. Firstly, the GenCo's objective model which is to maximise expected profit and to minimise risk of profit variation is solved by SPSO-TVAC. Secondly, the market clearing model which is to minimise system cost of locational marginal price (LMP) based market is solved by DC optimal power flow (DCOPF). The Monte Carlo method is employed to simulate other bidders' behaviour in competitive environment. Test results on the PJM 5-bus system indicate that SPSO-TVAC is superior to inertia weight approach particle swarm optimisation (IWAPSO) and genetic algorithm (GA) in searching the optimal trading solution. In addition, different bilateral contract prices and spot demand significantly impact GenCos' trading behaviour. Accordingly, the proposed approach could be a beneficial decision-making tool for a GenCo in energy trading.
\end{abstract}

Keywords: Optimal bidding strategy, energy trading, locational marginal price, bilateral contract market, particle swarm optimisation, Monte Carlo simulation.

\section{Introduction}

In energy market, generation companies (GenCos) should have strategies to maximise their profits and to avoid price risk (Shahidehpour et al., 2002). For example, GenCos may allocate their power into several markets including spot, bilateral contract or reserve markets. For trading in spot market, a GenCo could develop a bidding strategy to maximise its profit especially in imperfect competitive environment. In fact, spot price could be influenced by market participants' behaviour and demand fluctuation. Therefore, hedging price risk with low risk transaction such as bilateral contract is necessary for GenCos.

Recently, there are a number of researchers investigating optimal bidding strategies in locational marginal price (LMP) based markets. In Li and Shahidehpour (2005), a game-based bidding strategy for GenCos with incomplete information is proposed to find the supply function equilibrium. However, with the complexity of the problem, the global optimal solution is difficult to be found by this approach. In Gountis and Bakirtzis (2004), genetic algorithm (GA) and Monte Carlo (MC) simulation are applied to provide the optimal bidding strategy. Different risk profiles of market participants are also considered. However, only single period trading without technical constraints is considered. Finally, in Badri et al. (2008), a network-constrained bidding strategy considering bilateral contracts based on the primal dual interior point method (PDIPM) is proposed. It is assumed that all players' cost functions are known and the equilibrium point is provided. However, it is virtually impossible to know such confidential information.

With the complexity and large scale of the network-constraint bidding strategy problem, an efficient heuristic approach is needed to provide the optimal solution. In Bajpai and Singh (2008), fuzzy adaptive particle swarm optimisation (FAPSO) is proposed to solve the strategic bidding problem. The simulation results indicate that FAPSO is superior to GA in both searching performance and execution time. However, a proper design of the fuzzy set is needed to adapt the velocity weight for the particular problem. 
In Ratnaweera et al. (2004), self-organising hierarchical particle swarm optimisation with time-varying acceleration coefficients (SPSO-TVAC) is proposed. It is applied to a non-convex economic dispatch (Chaturvedi et al., 2008). The results show that SPSO-TVAC is efficient to solve a non-convex and non-differentiable function and its performance is superior to several other particle swarm optimisation (PSO). In addition, the velocity weight coefficient which is a key parameter of PSO is eliminated. Thus, it is applicable to solve various optimisation problems.

This paper proposes an optimal trading strategy for a GenCo in multi-market environment including day-ahead LMP-based and long term bilateral contract (Hou and $\mathrm{Wu}$, 2007) markets using a novel stochastic search technique, SPSO-TVAC. The trading strategy is formulated as a two-stage optimisation problem. The first subproblem is the GenCo's objective model, maximising expected profit from trading in the both markets and minimising risk of profit variation. The second is the market clearing process based on DCOPF to minimise system cost. MC simulation is employed to deal with the uncertainties from other bidders' behaviour in spot market. The proposed strategy is illustrated on the PJM 5-bus system and compared to the well-known stochastic search approaches, inertia weight approach particle swarm optimisation (IWAPSO) and GA.

The rest of the paper is organised as follows: Section 2 expresses problem formulation including the GenCo's objective model and the market clearing model. Section 3 describes the solution methodologies including SPSO-TVAC and MC simulation, and the solution algorithm. Section 4 illustrates the proposed strategy through numerical examples and discussions. Finally, Section 5 concludes the paper.

\section{Problem Formulation}

2.1 GenCo's objective model: To maximise the expected profit for a GenCo from spot and bilateral contract markets and to minimise risk of profit variation, the GenCo's objective model could be expressed as

$$
\begin{array}{ll}
\max & \sum_{t=1}^{T} \sum_{n=1}^{N}\left(\mathrm{E}\left[\eta_{n t}^{d}\right]+\eta_{n t}^{b}-\sigma_{n t}\right) \\
\text { s.t. } & q_{\min n} \leq q_{n t} \leq q_{\max n}, \quad q_{n t}=q_{n t}^{d}+q_{n t}^{b} \\
& \left|\Delta q_{n t}\right| \leq U R_{n}, \quad \text { if } \Delta q_{n t}>0 \\
& \left|\Delta q_{n t}\right| \leq D R_{n}, \quad \text { if } \Delta q_{n t}<0
\end{array}
$$

In (1), the multiple objectives of a GenCo are considered as the single objective function. Generally, for conflicting objectives, the tradeoff technique could also be implemented as $w_{1} \cdot\left(\mathrm{E}\left[\eta_{n t}^{d}\right]+\eta_{n t}^{b}\right)-w_{2} \cdot \sigma_{n t}$ (Boonchuay and Ongsakul, 2009). Here, it is assumed that all GenCo's objectives are equally weighted so that all weight coefficients could be set to one. The technical constraints, which are the maximum and minimum generation limits in (2), and ramping up/down limits in (3) and (4), are considered. The minimum up/down time constraints could also be included if some units need to be shut off.

The detail of the GenCo's objective model in (1) is written as

$$
\begin{aligned}
\mathrm{E}\left[\eta_{n t}^{d}\right] & =\frac{1}{M} \sum_{m=1}^{M}\left(\lambda_{m t}^{d} \cdot \tilde{q}_{n m t}^{d}-\frac{\tilde{q}_{n m t}^{d}}{\tilde{q}_{n m t}^{d}+q_{n t}^{b}} \cdot c_{n t}\left(\tilde{q}_{n m t}^{d}, q_{n t}^{b}\right)\right) \\
\eta_{n m t}^{d} & =\lambda_{m t}^{d} \cdot \tilde{q}_{n m t}^{d}-\frac{\tilde{q}_{n m t}^{d}}{\tilde{q}_{n m t}^{d}+q_{n t}^{b}} \cdot c_{n t}\left(\tilde{q}_{n n t}^{d}, q_{n t}^{b}\right) \\
\eta_{n t}^{b} & =\lambda_{t}^{b} \cdot q_{n t}^{b}-\frac{q_{n t}^{b}}{\bar{q}_{n t}^{d}+q_{n t}^{b}} \cdot c_{n t}\left(\bar{q}_{n t}^{d}, q_{n t}^{b}\right) \\
\sigma_{n t} & =\sqrt{\frac{1}{M} \sum_{m=1}^{M}\left(\eta_{n m t}^{d}-\mathrm{E}\left[\eta_{n t}^{d}\right]\right)^{2}}
\end{aligned}
$$

In (9) to (10), the cost function of unit $i$ at period $t$ is described as (Bajpai and Singh, 2007)

$$
\begin{aligned}
c_{n t}\left(q_{n t}\right) & =c_{0 n}+c_{1 n} \cdot q_{n t}+c_{2 n} \cdot q_{n t}^{2}+\left|c_{3 n} \cdot \sin \left(c_{4 n} \cdot\left(q_{\min n}-q_{n t}\right)\right)\right| \\
q_{n t} & =q_{n t}^{d}+q_{n t}^{b} .
\end{aligned}
$$


2.2 Market clearing model using DCOPF: In LMP-based market, the system operator generally provides electricity price with minimum system cost considering transmission limits. ACOPF is an efficient tool to determine electricity price and quantity for each bus. However, ACOPF requires a long execution time to obtain the optimal solutions. Therefore, DCOPF is used to provide market clearing price and quantity, which can be expressed as

$$
\begin{array}{ll}
\min & \sum_{i=1}^{I} p_{i} \cdot q_{i} \\
\text { s.t. } & \sum_{i=1}^{I} q_{i}-\sum_{i=1}^{I} L_{i}-P_{\text {Loss }}=0 \\
& F_{k} \leq \text { Limit }_{k}, \text { for } k=1,2, \ldots, K \\
& q_{\min i} \leq q_{i} \leq q_{\max i}, \text { for } i=1,2, \ldots, I
\end{array}
$$
Where the system loss $P_{\text {Loss }}$ is calculated by $\sum_{k=1}^{K} F_{k}^{2} \cdot R_{k}$, and the line flow $F_{k}$ is provided by $\sum_{i=1}^{I} G S F_{k-i} \cdot\left(q_{i}-L_{i}\right)($ Wood and
Wollenberg, 1996).

In the DCOPF model, the entire system loss will be compensated by the generator at the reference bus. Therefore, for more accurate LMP calculation, the fictitious nodal demand (FND) is included in the line flow equation as (Li and Bo, 2007)

$$
F_{k}=\sum_{i=1}^{I} G S F_{k-i} \cdot\left(q_{i}-L_{i}-E_{i}\right)
$$

In (15), $E_{i}$ is the FND at bus $i$, which can be calculated by

$$
E_{i}=\sum_{k=1}^{K_{i}} \frac{1}{2} \cdot \hat{F}_{k}^{2} \cdot R_{k}
$$

LMP at the reference bus is equal to the Lagrangian multiplier of the equality constraint in (12). For providing LMP at the other buses, the inequality constraint Lagrangian multiplier and the marginal loss factor have to be determined (Li and Bo, 2007).

\section{Solution Methodologies}

3.1 Self-organising hierarchical PSO with time-varying acceleration coefficients: SPSO-TVAC is an efficient population-based optimisation technique, which is appropriate for non-convex optimisation problems (Chaturvedi et al., 2008). Mathematically, it is defined as

$$
\begin{aligned}
& v_{i d}^{k+1}=a_{1}^{k} \cdot \operatorname{rand}_{1} \cdot\left(p_{i d}^{k}-x_{i d}^{k}\right)+a_{2}^{k} \cdot \operatorname{rand}_{2} \cdot\left(p_{g d}^{k}-x_{i d}^{k}\right) \\
& x_{i d}^{k+1}=x_{i d}^{k}+v_{i d}^{k+1} .
\end{aligned}
$$

In (17), the first component is the cognitive component that represents the individual experience of each particle. The last component is the social collaboration of the particles in finding the global optimal solution. The position of the $i$ th particle on the $d$ th dimension is updated by (18). With the TVAC concept, the acceleration coefficients are given as

$$
\begin{aligned}
& a_{1}^{k}=a_{1 i}-\frac{a_{1 i}-a_{1 f}}{k_{\max }} \cdot k \\
& a_{2}^{k}=a_{2 i}-\frac{a_{2 i}-a_{2 f}}{k_{\max }} \cdot k .
\end{aligned}
$$

In addition, SPSO-TVAC has a reinitialised process to enhance its performance when particles stagnate during the search. The pseudocode is shown as follows. 


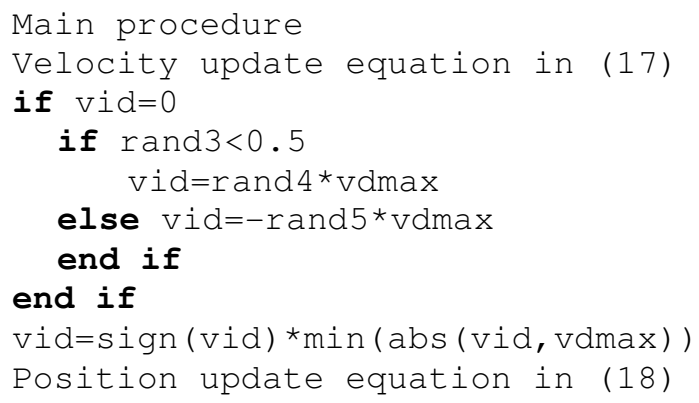

$v_{i d}$ is the velocity of the $i$ th particle on the $d$ th dimension, and $v_{d m a x}$ is the maximum velocity limit on the $d$ th dimension, which could be set as $10 \%$ of the dynamic range of the variable on each dimension (Chaturvedi et al., 2008).

3.2 Monte Carlo simulation for bidding strategy: MC simulation is a stochastic computational technique which is performed by statistical sampling experiments on a computer. It needs a probability density function (PDF) of uncertainty source to generate the sampling. For strategic bidding problems, the uncertainties come from bidding behaviour of market participants. A PDF that appropriately represents the distribution of historical bidding behaviour could be used. However, in this paper, a normal PDF is employed to estimate rivals' bidding behaviour, which can be expressed as (Bajpai and Singh, 2007)

$$
p d f\left(\tilde{p}_{n}^{r}\right)=\frac{1}{\sqrt{2 \pi} \sigma_{n}^{r}} \cdot \exp \left(-\frac{\left(\tilde{p}_{n}^{r}-\mu_{n}^{r}\right)^{2}}{2\left(\sigma_{n}^{r}\right)^{2}}\right) .
$$

3.3 Solution algorithm: The proposed solution algorithm based on PSO and MC simulation for the strategic trading problem is described in 10 steps as shown in Figure 1.

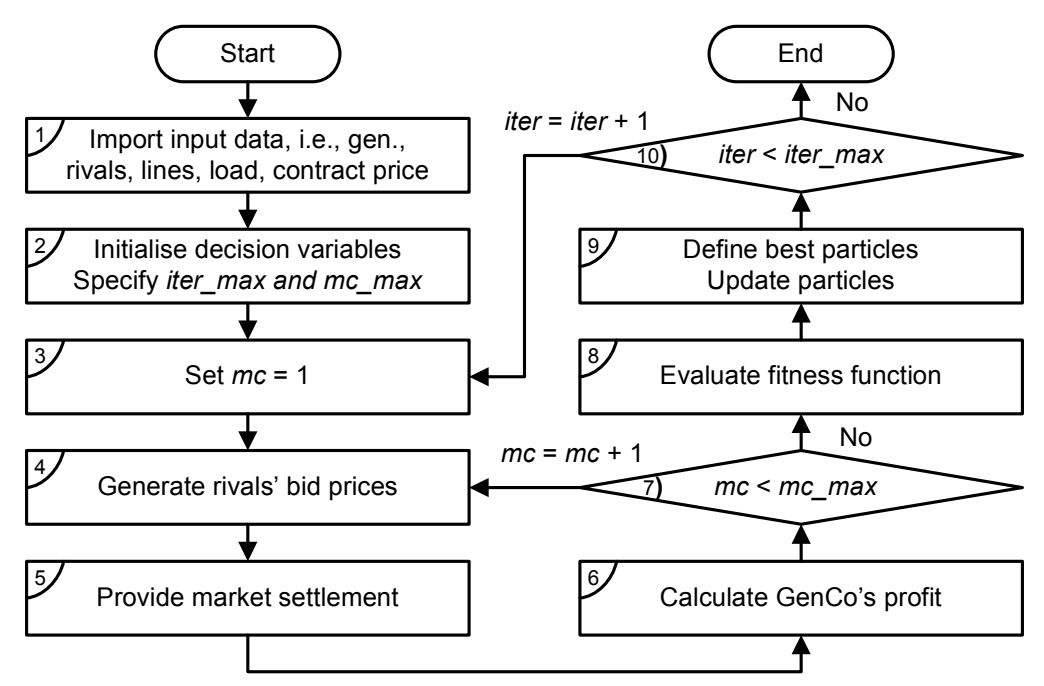

Figure 1. Solution procedure

Step 1) Input data: The concerned GenCo's cost data, historical rivals' bidding data, transmission lines, load, and bilateral contract price are given.

Step 2) Initial value: Decision variables including bid price and quantity, and amount of bilateral transaction are randomly initialised. The maximum number of PSO iterations and MC trials are specified.

Step 3) Reset MC counter: The MC counter is set/reset to be 1.

Step 4) Rivals' strategy estimation: The rivals' bid prices are generated based on their PDF.

Step 5) Market settlement process: The DCOPF model in (11) to (16) is solved by a linear programming approach to provide LMP and dispatched quantity for each bus. 
Step 6) Profit evaluation: GenCo's profit from trading in spot market is calculated using (6). This finishes one trial of MC simulation.

Step 7) MC loop stopping criteria: If the maximum number of the simulation is not reached, go to Step 4 and the MC counter is increased by 1 . Otherwise go to the next step.

Step 8) Evaluating fitness value: The fitness function including the objective function in (1) and the penalty function (PF) is evaluated by

$$
\text { Fitness }=\sum_{t=1}^{T} \sum_{n=1}^{N}\left(\mathrm{E}\left[\eta_{n t}^{d}\right]+\eta_{n t}^{b}-\sigma_{n t}\right)-P F
$$

where $P F$ is a constant that is larger than zero if the constraints in (2) to (4) are not satisfied. Otherwise $P F$ equals to 0.

Step 9) Updating particles: The best fitness particles, global and local best particles, are defined and all particles are updated by (17) and (18).

Step 10) PSO loop stopping criteria: If the PSO counter is less than maximum number of the PSO iterations, go to Step 3 and increase the PSO counter by 1 . Otherwise stop.

\section{Numerical Example and Discussion}

The proposed optimal trading strategy is tested on the PJM 5-bus system. The optimisation problem in (1) to (4) is solved by different optimisation techniques including SPSO-TVAC, IWAPSO, and GA. In addition, the DCOPF model in (11) to (16) is a suboptimisation problem. As the simulation is performed on the MATLAB environment, the DCOPF model is conveniently solved by 'fmincon' function included in the software package. To reduce the computing effort, an artificial neural network (ANN) is used to approximate the LMP. The simulation is performed on a $2.8 \mathrm{GHz}$ Pentium IV personal computer with 1GB RAM.

The configuration and parameters of the PJM 5-bus system are shown in Figure 2 and Table 1, respectively. There are four GenCos participating in the market. In this example, the concerned GenCo, GenCo-A, needs to provide the optimal bid price, bid quantity, and amount of bilateral transaction to maximise expected profit and to minimise risk of profit variation. Therefore, the rest GenCos are the opponent bidders and the bidding data is given in Table 2.

The production cost function of GenCo-A is expressed in (9). The cost coefficients and the technical parameters are given in Table 3.

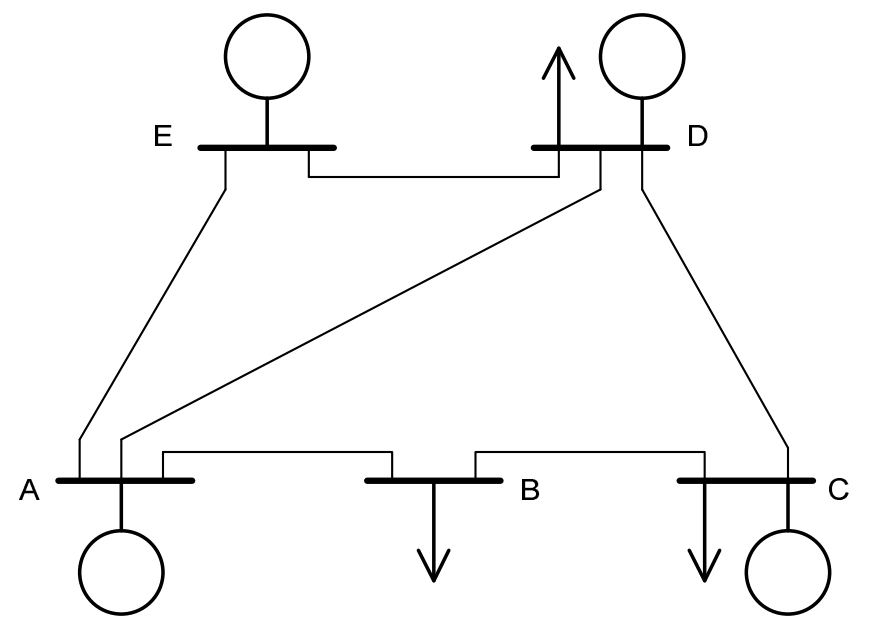

Figure 2. PJM 5-bus system

Table 1. Transmission line data of PJM 5-bus system

\begin{tabular}{|c|c|c|c|c|c|c|}
\hline & A-B & A-D & A-E & B-C & C-D & D-E \\
\hline $\mathrm{R}(\%)$ & 0.281 & 0.304 & 0.064 & 0.108 & 0.297 & 0.297 \\
\hline $\mathrm{X}(\%)$ & 2.810 & 3.040 & 0.640 & 1.080 & 2.970 & 2.970 \\
\hline Limit & 999 & 999 & 999 & 999 & 999 & 240 \\
\hline
\end{tabular}


Table 2. Rivals' bidding data

\begin{tabular}{|c|c|c|c|}
\hline GenCo & $\begin{array}{c}\text { Mean of bid price, } \\
\$ / \mathrm{MWh}\end{array}$ & $\begin{array}{c}\text { SD. of bid price, } \\
\$ / \mathrm{MWh}\end{array}$ & Bid quantity, MW \\
\hline $\mathrm{C}$ & 30 & 4 & 520 \\
\hline $\mathrm{D}$ & 35 & 5 & 200 \\
\hline $\mathrm{E}$ & 10 & 3 & 600 \\
\hline
\end{tabular}

Table 3. GenCo-A's data

\begin{tabular}{|c|c|c|c|c|c|c|c|c|}
\hline$c_{0}$ & $c_{1}$ & $c_{2}$ & $c_{3}$ & $c_{4}$ & $\begin{array}{c}q_{\max }, \\
\text { MW }\end{array}$ & $\begin{array}{c}q_{\min }, \\
\text { MW }\end{array}$ & $\begin{array}{c}U R, \\
\text { MW }\end{array}$ & $\begin{array}{c}D R, \\
\text { MW }\end{array}$ \\
\hline 0.00482 & 7.97 & 78 & 150 & 0.063 & 210 & 20 & 30 & 50 \\
\hline
\end{tabular}

In the simulation, it is assumed that there is no demand elasticity and each load bus equally shares the spot demand. In addition, there is a large customer at Bus-D who prefers to purchase power from GenCo-A through bilateral transaction at specific prices and the bilateral congestion charge is ignored.

4.1 Solution convergence comparison: Three different global search approaches including SPSO-TVAC, IWAPSO, and GA are employed to provide the optimal trading strategy. For the PSO parameters, the maximum number of iterations is taken as 300 with the swarm size of 20 particles. For SPSO-TVAC, the coefficient of cognitive component $\left(a_{1}\right)$ is decreased in the range of 2.5 to 0.5 and the social learning factor $\left(a_{2}\right)$ is increased from 0.5 to 2.5. For IWAPSO, the inertia weight is varied from 0.9 to 0.4 and the acceleration coefficients are equal to 2 . For the GA parameters, the maximum number of generation, population size, and mutation and crossover probabilities are used as $300,20,0.2$, and 0.6 , respectively.

The single period trading with spot demand of $900 \mathrm{MW}$ and bilateral contract price of $\$ 10 / \mathrm{MWh}$ is used to compare the performances of the optimisers in the strategic trading problem. The 100-trial MC simulation is performed to simulate the rivals' behaviour. By 20 runs of the simulation, the convergence characteristics of the best solutions from SPSO-TVAC, IWAPSO, and GA are compared in Figure 3.

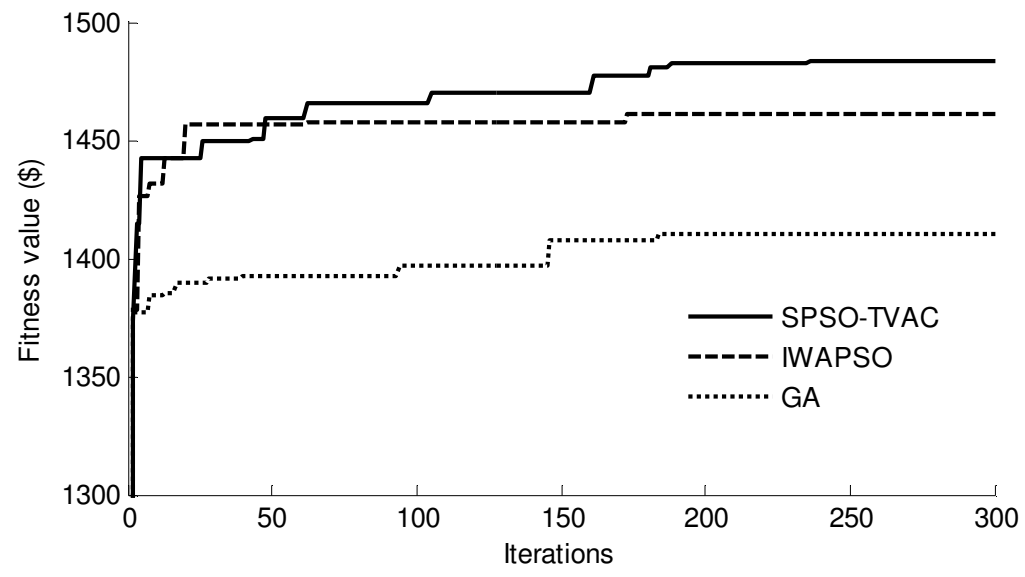

Figure 3. Convergence characteristics of different optimisers

In the beginning of the search, the SPSO-TVAC and IWAPSO fitness values dramatically increase, while the GA fitness value slightly increases. After the 50th iteration, SPSO-TVAC provides higher fitness solutions than IWAPSO and GA. An advantage of SPSO-TVAC is that the reinitialised process will be active when a particle stagnates during the search. Therefore, all particles are always encouraged to find a better solution. At the last iteration, the SPSO-TVAC fitness value is $\$ 1,478.8$, whereas the IWAPSO and GA fitness values are $\$ 1,461.2$ and $\$ 1,410.5$, respectively. For the execution time, the PSO-based approaches require approximately 60 minutes, but the GA approach needs around 80 minutes to provide the optimal solution.

4.2 LMP approximation using ANN: A feed-forward ANN with 15 hidden nodes and 3 hidden layers is used to find LMP. The ANN has been trained by 30,000 input/output patterns from the DCOPF solutions. The ANN diagram for the LMP approximation is shown in Figure 4. In this case, a single period trading with spot demand of $900 \mathrm{MW}$ and bilateral contract price of $\$ 10 / \mathrm{MWh}$ is considered. SPSO-TVAC is employed to maximise the GenCo's objective model. The solutions from the DCOPF and the ANNbased LMP approximations are shown in Table 4. 


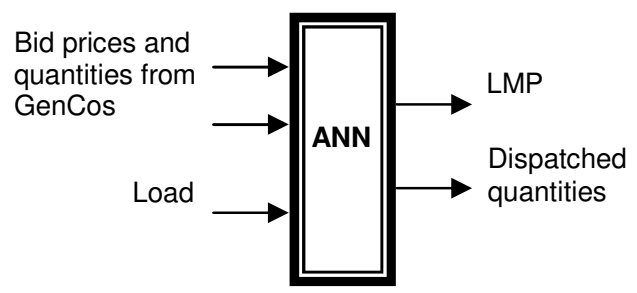

Figure 4. Diagram of the ANN-based LMP approximation

Table 4. Comparative results from different LMP approximation approaches

\begin{tabular}{|l|r|r|r|r|}
\hline & \multicolumn{1}{|c|}{100 trials of MC } & \multicolumn{2}{c|}{500 trials of MC } \\
\cline { 2 - 5 } & DCOPF & ANN & DCOPF & \multicolumn{1}{c|}{ ANN } \\
\hline Bid price, \$/MWh & 15.26 & 16.54 & 15.48 & 16.65 \\
\hline Bid quantity,MW & 84.38 & 83.84 & 84.46 & 83.83 \\
\hline Bilateral transaction, MW & 115.43 & 115.64 & 114.95 & 115.67 \\
\hline Expected LMP at Bus-A, \$/MWh & 29.68 & 29.67 & 29.60 & 29.65 \\
\hline Expected profit from spot,\$ & 1716.52 & 1705.54 & 1713.16 & 1703.51 \\
\hline Profit from bilateral,\$ & 76.18 & 78.35 & 77.65 & 78.37 \\
\hline Risk of profit variation,\$ & 313.81 & 324.64 & 332.18 & 327.49 \\
\hline Execution time, min & 53.12 & 2.51 & 255.48 & 14.35 \\
\hline Computer Configuration: $2.8 \mathrm{GHz}$, PIV Processor, 1GB RAM \\
\hline
\end{tabular}

In Table 4, the decision variables of GenCo-A are bid price, bid quantity and amount of bilateral transaction. The other parameters are state variables. For the ANN approach with 100-trial MC simulation, on average, the decision variables differ $3.06 \%$ from the DCOPF solutions while the other parameters differ $1.74 \%$. Using 100-trial MC simulation, the ANN approach requires 2.51 minutes, whereas the DCOPF approach needs 53.12 minutes to provide the optimal solution. For the ANN approach with 500-trial MC simulation, the decision and the state variables differ from the DCOPF solutions $2.98 \%$ and $0.76 \%$, respectively. Using 500-trial MC simulation, the execution times of the ANN and the DCOPF approaches are 14.35 minutes and 255.48 minutes, respectively. The results indicate that the ANN approach could significantly reduce the computing effort in providing the optimal trading strategy.

Note a higher number of MC simulation could provide more accurate solutions. However, it is necessary to consider the execution time. When MC simulation is taken as 100 trials, the execution time is reduced by 5 times, but the solutions are not as diverse as the 500-trial solutions.

4.3 Different bilateral contract prices: In this case, the optimal trading strategies for GenCo-A with different bilateral contract prices are illustrated. The bilateral contract price at Bus-D varies from $\$ 15$ to $\$ 28 / \mathrm{MWh}$ with the spot demand of $900 \mathrm{MW}$. The optimal solutions are shown in Table 5.

Table 5. Optimal solutions with different bilateral contract prices

\begin{tabular}{|l|r|r|r|r|}
\hline & \multicolumn{4}{|c|}{ Bilateral contract price \$/MWh } \\
\cline { 2 - 5 } & 15 & 20 & \multicolumn{1}{|c|}{25} & \multicolumn{1}{c|}{28} \\
\hline Bid price, \$/MWh & 16.94 & 16.44 & 15.50 & 15.28 \\
\hline Bid quantity, MW & 83.83 & 83.69 & 80.42 & 2.26 \\
\hline Bilateral transaction, MW & 115.66 & 126.22 & 129.56 & 207.24 \\
\hline Expected LMP at Bus-A, \$/MWh & 29.67 & 29.62 & 29.62 & 29.49 \\
\hline Expected profit from spot, \$ & 1705.31 & 1660.93 & 1594.36 & 54.54 \\
\hline Profit from bilateral, \$ & 656.69 & 1288.54 & 1970.42 & 3773.51 \\
\hline Risk of profit variation, \$ & 324.47 & 324.44 & 311.20 & 18.48 \\
\hline
\end{tabular}

In Table 5, the optimal solutions for GenCo-A with different bilateral contract prices are compared. The bid price and the expected LMP at Bus-A are quite stable even the bilateral contract price varies. Furthermore, the relation between the allocated power in each market and the bilateral contract price is non-linear as shown in Figure 5. When the bilateral contract price is less than $\$ 24 / \mathrm{MWh}$, GenCo-A keeps the same amount of allocated power in each market. However, when the bilateral contract price increases to $\$ 27 / \mathrm{MWh}$, GenCo-A might allocate the entire power to the bilateral contract market. 


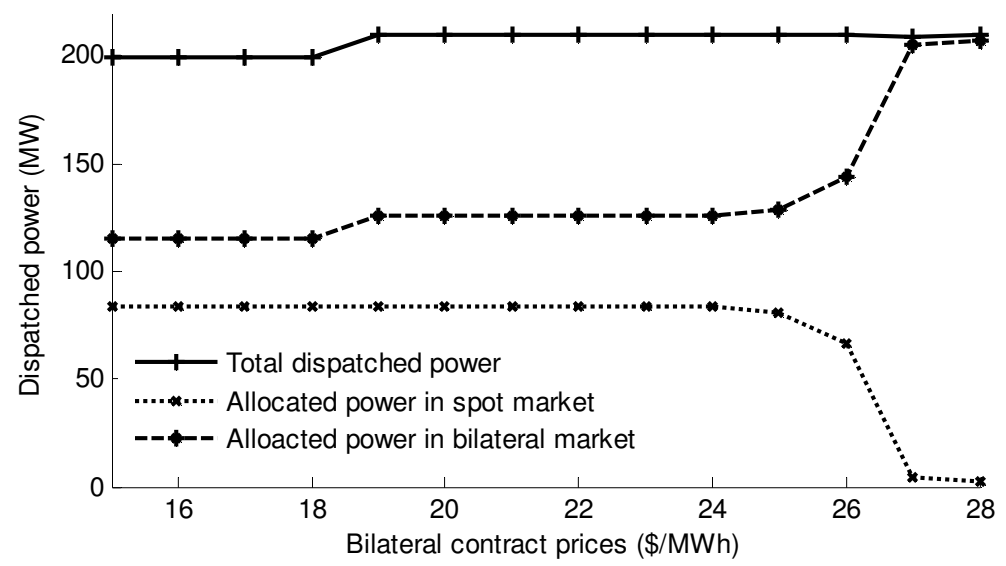

Figure 5. GenCo-A's trading behaviour versus different bilateral contract prices

In Figure 6, GenCo-A's expected profit and risk of profit variation from spot market, and profit from bilateral transaction with different bilateral contract prices are shown. Varying the bilateral contract prices from $\$ 15$ to $\$ 24 / \mathrm{MWh}$, the expected profit is not diverse because the expected LMP and dispatched quantity at Bus-A are rather flat as shown in Table 5. In bilateral contract market, as the price increases, the profit is linearly growing. Nevertheless, when the bilateral contract price is higher than $\$ 25 / \mathrm{MWh}$, profit from the both markets will sharply changes. The risk correlates with the expected profit from spot market trading, which is close to zero when the bilateral contract price is equal to $\$ 28 / \mathrm{MWh}$.

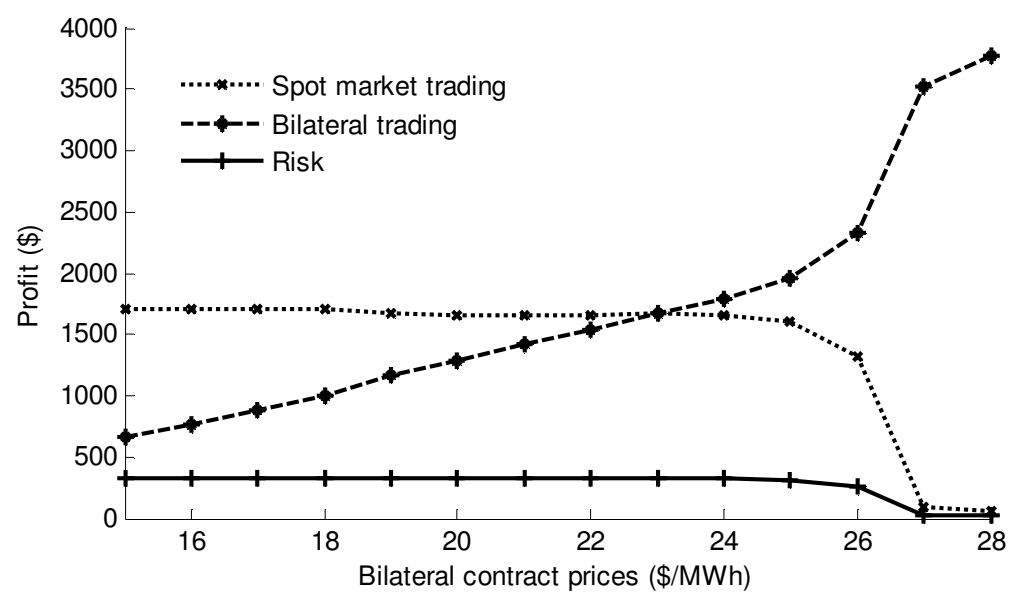

Figure 6. Profit and risk of GenCo-A versus different bilateral contract prices

4.4 Multi-hourly trading strategy: Day-ahead spot and bilateral contract markets are considered to provide the optimal bid price and power allocation for GenCo-A. The day-ahead spot demand is shown in Figure 7. It is assumed that the bilateral contract price at Bus-D is $\$ 15 / \mathrm{MWh}$ for the entire trading periods.

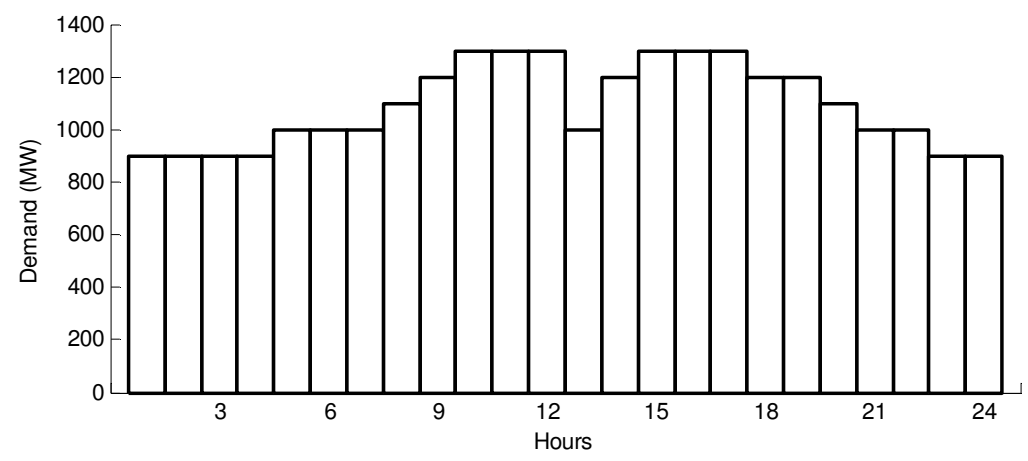

Figure 7. Forecast demand for day-ahead spot market 
In Figure 8, the allocated power in spot and bilateral contract markets and total dispatched power of GenCo-A for 24-hour trading period are shown. The results indicate that different spot demands cause strategy changes of GenCo-A. However, in entire trading periods, GenCo-A allocates a higher energy proportion in bilateral contract market to hedge price risk. The average dispatched output of GenCo-A is 199.58 MW. With the different demands, dispatched levels of GenCos vary as shown in Table 6.

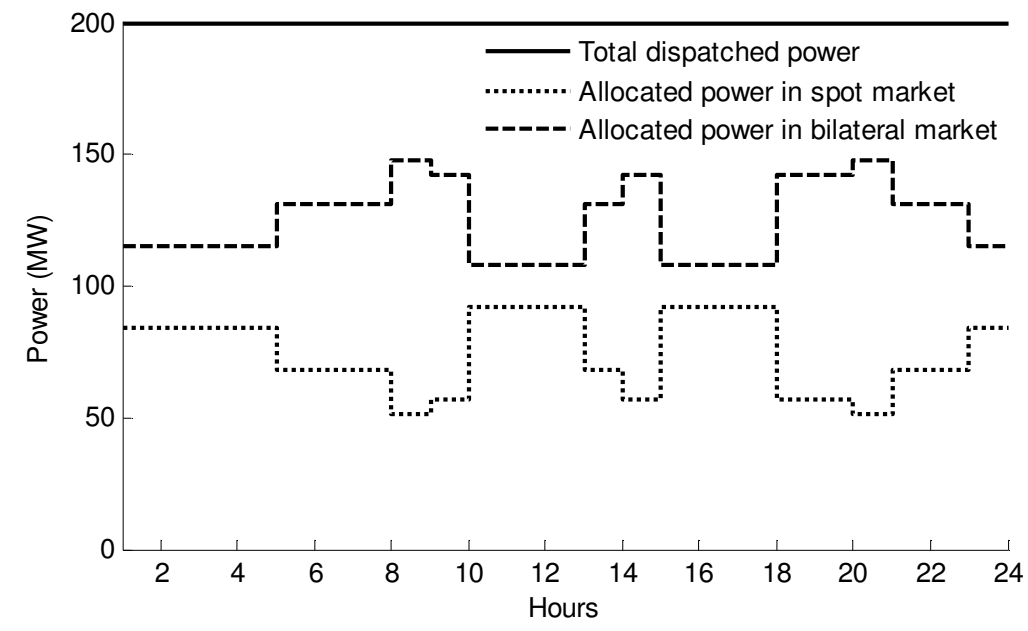

Figure 8. Total dispatch and allocated power of GenCo-A in day-ahead spot and bilateral contract markets

Table 6. Expected power dispatch of each GenCo with different spot demands

\begin{tabular}{|c|r|r|r|r|r|}
\hline \multirow{2}{*}{$\begin{array}{l}\text { GenCo } \\
\text { dispatch, MW }\end{array}$} & \multicolumn{5}{|c|}{ Spot demand, MW } \\
\cline { 2 - 6 } & 900 & 1000 & 1100 & 1200 & 1300 \\
\hline A & 84.39 & 68.13 & 51.84 & 57.23 & 91.78 \\
\hline C & 186.35 & 300.88 & 415.40 & 486.86 & 499.71 \\
\hline D & 35.41 & 36.72 & 38.20 & 61.44 & 114.60 \\
\hline E & 600.00 & 600.00 & 600.00 & 600.00 & 600.00 \\
\hline
\end{tabular}

In Figure 9, the expected LMP at Bus-A and the optimal bid price of GenCo-A for 24-hour trading period are shown. During peak demand periods of $1300 \mathrm{MW}$, the expected LMP at Bus-A increases to $\$ 34.94 / \mathrm{MWh}$, whereas the lowest LMP, with spot demand of $900 \mathrm{MW}$, is $\$ 29.68 / \mathrm{MWh}$. The optimal bid prices of GenCo-A are, on average, 58.11\% lower than the LMPs.

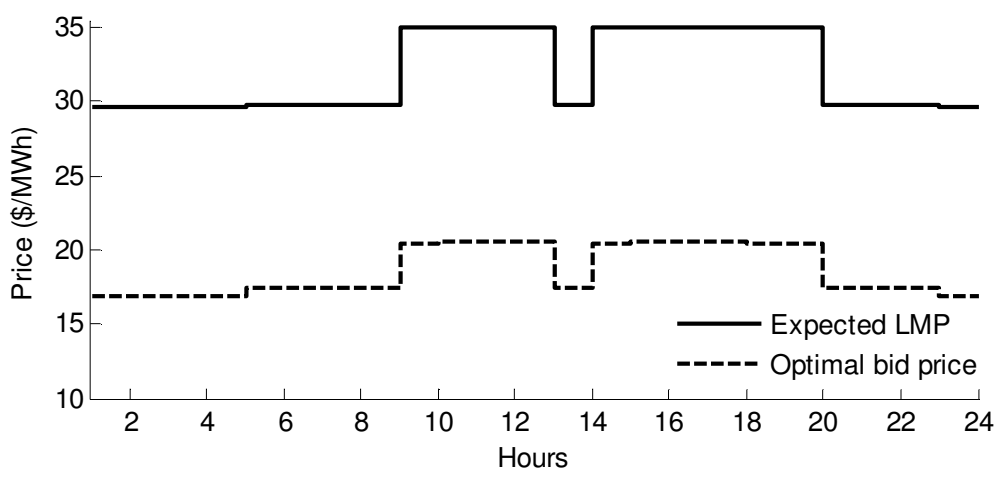

Figure 9. Optimal bid price and expected LMP at Bus-A

In Figure 10, the expected profit and risk of profit variation from spot market, and profit from bilateral transaction of GenCo-A for 24-hour trading period are shown. For all trading periods, the cumulative expected profit and risk of profit variation from spot market are $\$ 40,722$ and $\$ 7,060$, respectively. Profit from bilateral trading depends on amount of sold energy, which the cumulative bilateral profit is equal to $\$ 16,969$. 


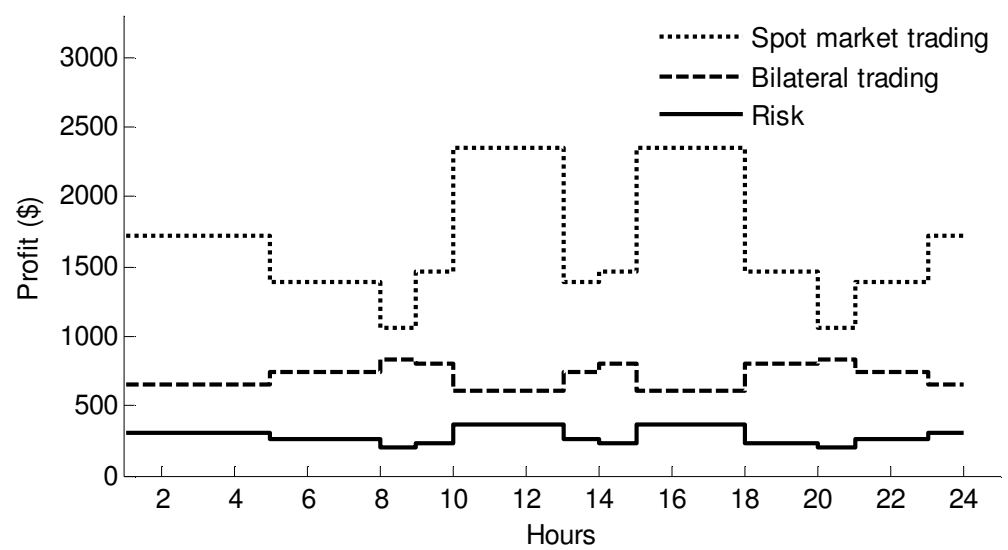

Figure 10. Profit and risk of GenCo-A in day-ahead spot and bilateral contract markets

\section{Conclusion}

In this paper, SPSO-TVAC and MC simulation are used to provide the optimal trading strategies for a GenCo in LMP-based and long term bilateral contract markets. The proposed trading strategy includes two optimisation stages. The first stage is the GenCo's objective model, which is to maximise expected profit in spot and bilateral contract markets and to minimise risk of profit variation, solved by SPSO-TVAC. The second stage is the market clearing model based on DCOPF to provide the LMP. The uncertainties from other bidders' behaviour are estimated by the MC approach. Test results indicate that SPSO-TVAC could provide a better solution than IWAPSO and GA in the strategic trading problem. The ANN-based LMP approximation approach is effective to reduce the computing effort in the optimisation process. With different bilateral contract prices and spot demands, the proposed approach could be used as a decision-making tool for a GenCo in energy trading.

\section{Nomenclature}

$a_{1}^{k}, a_{2}^{k} \quad$ Acceleration coefficients of cognitive and social components at iteration $k$, respectively.

$a_{1 i}, a_{2 i} \quad$ Initial values of cognitive and social acceleration coefficients, respectively.

$a_{1 f}, a_{2 f} \quad$ Final values of cognitive and social acceleration coefficients, respectively.

$c_{0 n}, c_{1 n}, c_{2 n}$ Production cost coefficients of unit $n$.

$c_{3 n}, c_{4 n} \quad$ Constants of the valve point loading effect of unit $n$.

$c_{n t}(\cdot) \quad$ Production cost function of unit $n$ at period $t(\$)$.

$D R_{n} \quad$ Ramping down limit of unit $n$ (MW).

$\mathrm{E}\left[\eta_{n t}^{d}\right] \quad$ Expected profit from trading in spot market of unit $n$ at period $t(\$)$.

$F_{k} \quad$ Line flow at line $k(\mathrm{MW})$.

$\hat{F}_{k} \quad$ Line flow at line $k$ from the previous iteration of the DCOPF calculation.

$G S F_{k-i} \quad$ Generator shift factor of line $k$ and bus $i$.

I Number of buses.

$k \quad$ Iteration index.

$k_{\max } \quad$ Maximum number of iterations.

$K \quad$ Number of transmission lines.

$L_{i} \quad$ Spot market load at bus $i$ (MW).

Limit $_{k} \quad$ Line flow limit at line $k(\mathrm{MW})$.

$M \quad$ Maximum number of rivals' strategies.

$N \quad$ Number of generation units of the concerned GenCo.

$p_{i} \quad$ Bid price at bus $i(\$ / \mathrm{MWh})$.

$\tilde{p}_{n}^{r} \quad$ Bid price of unit $n$ of rival $r$.

$p_{i d}^{k} \quad$ Best position of particle $i$ on dimension $d$ reached at iteration $k$.

$p_{g d}^{k} \quad$ Global best particle on dimension $d$ reached at iteration $k$.

$P_{\text {Loss }} \quad$ System loss (MW).

$q_{i} \quad$ Generation dispatch at bus $i$ (MW).

$q_{n t} \quad$ Generation output of unit $n$ at period $t(\mathrm{MW})$.

$q_{\max i} \quad$ Maximum generation dispatch at bus $i$ (MW). 


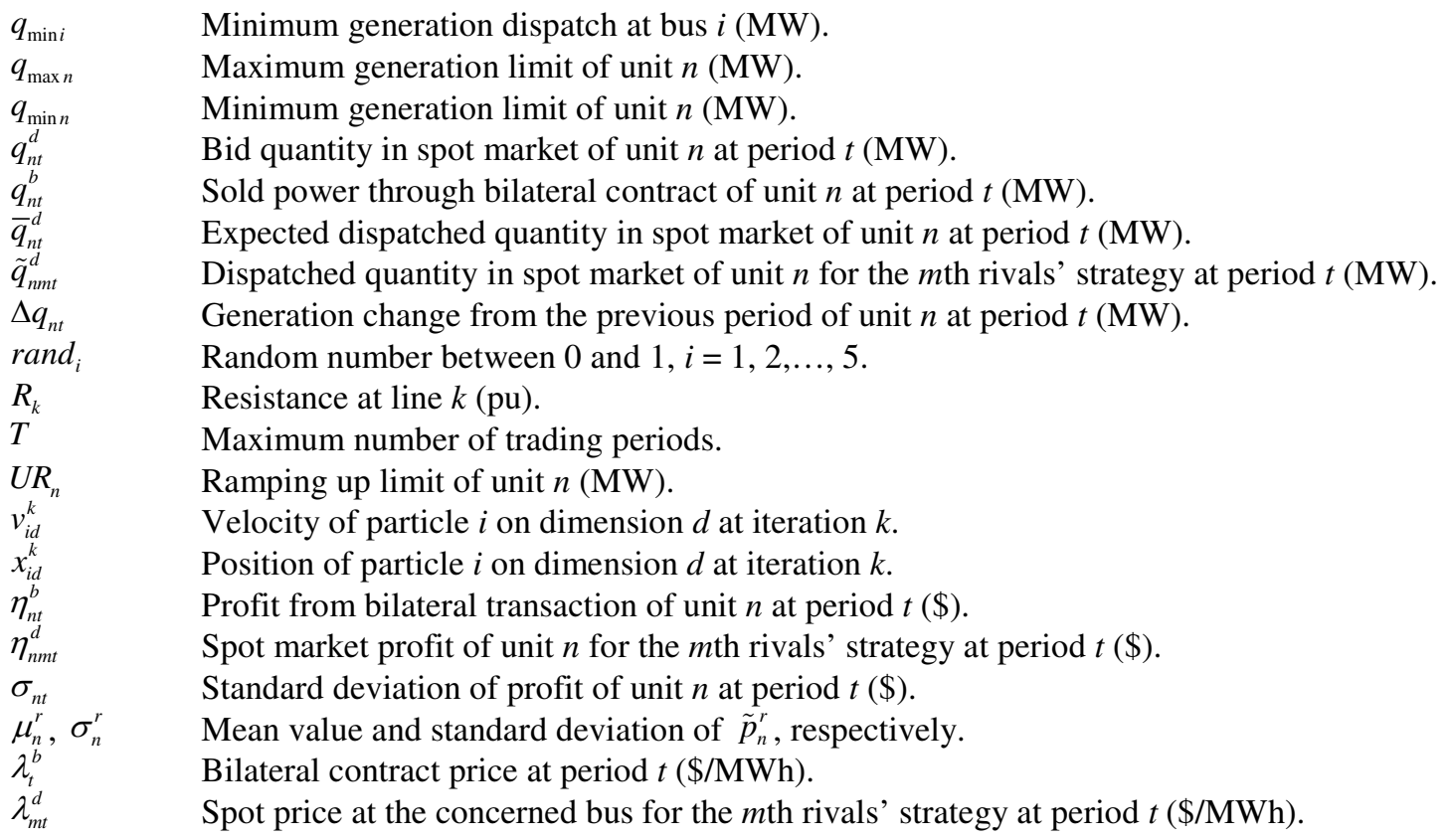

\section{Acknowledgement}

This research is supported by the Office of the Higher Education Commission, under the Ministry of Education, Thailand, and the Energy Policy and Planning Office (EPPO), under the Ministry of Energy, Thailand.

\section{References}

Badri A., Jadid S., Rashidinejad M. and Moghaddam M.P., 2008. Optimal bidding strategies in oligopoly markets considering bilateral contracts and transmission constraints. Electric Power Systems Research, Vol. 78, No. 6, pp. 1089-1098.

Bajpai P. and Singh S.N., 2007. Fuzzy adaptive particle swarm optimization for bidding strategy in uniform price spot market. IEEE Transactions on Power Systems, Vol. 22, No. 4, pp. 2152-2160.

Bajpai P. and Singh S.N., 2008. Strategic bidding in network constrained electricity markets using FAPSO. International Journal of Energy Sector Management, Vol. 2, No. 2, pp. 274-296.

Boonchuay C. and Ongsakul W., 2009. A Risk-Constrained Optimal Bidding Strategy for a Generation Company by IWAPSO. Proceedings of IEEE Bucharest PowerTech, Romania.

Chaturvedi K.T., Pandit M. and Srivastava L., 2008. Self-organizing hierarchical particle swarm optimization for nonconvex economic dispatch. IEEE Transactions on Power Systems, Vol. 23, No. 3, pp. 1079-1087.

Gountis V.P. and Bakirtzis A.G., 2004. Bidding strategies for electricity producers in a competitive electricity marketplace. IEEE Transactions on Power Systems, Vol. 19, No. 1, pp. 356-365.

Hou Y. and Wu F.F., 2007. Long-term bilateral contract pricing with risks of congestion charge. Proceedings of IEEE PES General Meeting, pp 1-6.

Li F. and Bo R., 2007. DCOPF-based LMP simulation: Algorithm, comparison with ACOPF, and sensitivity. IEEE Transactions on Power Systems, Vol. 22, No. 4, pp. 1475-1485.

Li T. and Shahidehpour M., 2005. Strategic bidding of transmission-constrained GENCOs with incomplete information. IEEE Transactions on Power Systems, Vol. 20, No. 1, pp. 437-447.

Optimization toolbox, available at: www.mathworks.com.

PJM Training Materials—LMP 101, available at: www.pjm.com.

Ratnaweera A., Halgamuge S.K. and Watson H.C., 2004. Self-organizing hierarchical particle swarm optimizer with time-varying acceleration coefficients. IEEE Transactions on Evolutionary Computation, Vol. 8, No. 3, pp. 240-255.

Shahidehpour M., Yamin H. and Li Z., 2002. Market Operations in Electric Power Systems. Wiley Interscience, New York. Wood A.J. and Wollenberg B.F., 1996. Power Generation, Operation, and Control. 2nd edition, Wiley Interscience, New York. 


\section{Biographical notes}

Chanwit Boonchuay received the M.Eng. degree in electrical engineering from King Mongkut's Institute of Technology North Bangkok, Thailand, in 2005. He is currently pursuing the D.Eng. degree in electric power system management at Asian Institute of Technology, Thailand. He spent two months as a visiting scholar at the University of Hong Kong in 2009. At present, he is a visiting scholar at the University of Tennessee, Knoxville. His current interests include power system optimisation, AI applications to power systems, and power system restructuring and deregulation.

Weerakorn Ongsakul received the M.S. and Ph.D. degrees in electrical engineering from Texas A\&M University, College Station, in 1991 and 1994, respectively. $\mathrm{He}$ is currently an Associate Professor of Energy and Dean of School of Environment, Resources, and Development, Asian Institute of Technology, Thailand. His current interests are in parallel processing applications, power system optimisation, AI applications to power systems, and power system restructuring and deregulation.

Jin Zhong received the B.Sc. degree from Tsinghua University, Beijing, China, in 1995, the M.Sc. degree from China Electric Power Research Institute, Beijing, in 1998, and the Ph.D degree from Chalmers University of Technology, Gothenburg, Sweden, in 2003. At present, she is an Assistant Professor in the Department of Electrical and Electronic Engineering at the University of Hong Kong. Her areas of interest are electricity sector deregulation, ancillary service pricing, and power system planning.

Felix F. Wu received the Ph.D. degree from the University of California at Berkeley (UCB). He is currently the holder of Philip K H Wong Wilson K L Wong Professor in Electrical Engineering and Chair Professor of Electrical Engineering at the University of Hong Kong (HKU). He is emeritus Professor of UCB and Director of Center for Electrical Energy System at HKU and Director of HKU-Tsinghua Shenzhen Power System Research Institute in Shenzhen, China. His research interests are electric energy industry restructuring, power system investment planning, design of modern control centers, distribution automation, distributed processing.

Received October 2009

Accepted February 2010

Final acceptance in revised form March 2010 